\title{
OTTV (SNI 03-6389-2011) and ETTV (BCA 2008) Calculation for Various Building's Shapes, Orientations, Envelope Building Materials: Comparison and Analysis
}

\author{
Loekita, S. ${ }^{1}$ and Priatman, J.2*
}

\begin{abstract}
The Indonesian National Standard SNI 03-6389-2000 adapted the 1983 Singapore's Handbook on Energy Conservation and limited the Overall Thermal Transfer Value (OTTV) of the building envelope to $45 \mathrm{Watt} / \mathrm{m}^{2}$. In 2008, the Singapore's Building and Construction Authority (BCA) shifted to Envelope Thermal Transfer Value (ETTV) value of $50 \mathrm{Watt} / \mathrm{m}^{2}$, while SNI 03-6389-2011 continues to use OTTV. This paper reviewed the new SNI 03-6389-2011 and compared it with BCA by calculating OTTV and ETTV of prismatic buildings with eight different shapes and building orientations, 11 Window to Wall Ratio, and 27 building envelope materials. This study also tested those variables to find the best building shape and orientation for an energy saver building. The result shows that ETTV (BCA) is stricter than OTTV (SNI 036389-2011) except the OTTV with black building envelope, while parallelogram shape building with North-South orientation is the best combination of energy saver building.
\end{abstract}

Keywords: Building shape; building orientation; envelope building material; ETTV; OTTV.

\section{Introduction}

People started to pay attention on energy conservation due to increasingly high demand of energy, especially in residential and commercial building sectors. Specifically in Singapore and Indonesia, air conditioning uses most energy in commercial buildings. Therefore, it is essential to understand the condition of air system when designing a building since the amount of energy usage must be established early on [1].

Energy consumption in Indonesia is regulated by limiting the Overall Thermal Transfer Value (OTTV) [2,3], adopting the Handbook on Energy Conservation in Buildings and Building Services published by the Development \& Building Control Division of Public Works Department of Singapore in 1983 [4]. In the meantime the new regulation in Singapore published by the Building and Construction Authority (BCA) [5] uses Envelope Thermal Transfer Value (ETTV) instead of OTTV. According to BCA [5] the later research works revealed that OTTV did not reflect accurately the relative performance of the different elements in an envelope system.

${ }^{1}$ Department of Civil Engineering, Faculty of Civil Engineering and Planning Petra Christian University, Surabaya, INDONESIA

${ }^{2}$ Department of Architecture, Faculty of Civil Engineering and Planning Petra Christian University, Surabaya, INDONESIA.

* Corresponding author; e-mail: jpriatman@petra.ac.id

Note: Discussion is expected before November $1^{\text {st }} 2015$, and will be published in the "Civil Engineering Dimension" volume 18, number 1, March 2016.

Received 24 February 2015; revised 12 July 2015; accepted 17 August 2015.
The difference between OTTV [2,3] and ETTV [5] is in the limiting value and the level of sun radiation absorption. The OTTV in SNI 03-6389-2011 is limited to $35 \mathrm{~W} / \mathrm{m}^{2}$ and considers sun radiation absorption (a) due to the color of building envelope in the formulation. While the limiting ETTV value according to $\mathrm{BCA}$ is $50 \mathrm{~W} / \mathrm{m}^{2}$, and no sun radiation absorption coefficient $\alpha$. in the formulation.

\section{Concept of OTTV}

The foundation of an energy efficient building starts with its design process. The main issue in creating an energy efficient building comes from the absorption of the building's solar heat load through its air conditioning system. Aligning the direction of the building's façade to East and West and choosing light colors for wall finish are some examples of the common design practice to reduce solar heat input. Limiting OTTV is one of the energy efficiency strategies. OTTV takes into account the elements of heat gain through the external wall of a building, such as: heat conduction through opaque walls and glass windows as well as solar radiation through glass windows. OTTV value is measured by taking the average measurement of these three elements over the whole envelope area of the building.

\section{OTTV based on SNI 03-6389-2011[3]}

To calculate the OTTV of an external wall, the following basic equations shall be used:

$\mathrm{OTTV}=\alpha\left[\mathrm{U}_{\mathrm{w}}(1-\mathrm{WWR}) \mathrm{TD}_{\mathrm{ek}}\right]+\left(\mathrm{U}_{\mathrm{f}} \mathrm{WWR} \Delta \mathrm{T}\right)+(\mathrm{SC}$ WWR SF) 
Where more than one type of material and/or fenestration is used, the following equation shall be used:

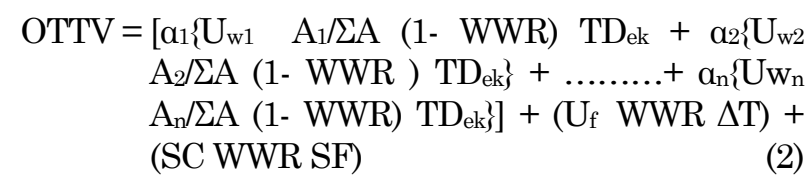

While to calculate the OTTV for the envelope of the whole building, the following equation shall be used:

$$
\begin{aligned}
& \text { OTTV }= \\
& \frac{\left(\mathrm{A}_{01} \mathrm{OTTV}_{01}\right)+\left(\mathrm{A}_{02} \mathrm{OTTV}_{02}\right)+\ldots \ldots .+\left(\mathrm{A}_{0 \mathrm{i}} \mathrm{OTTV}_{0 \mathrm{i}}\right)}{\mathrm{A}_{01}+\mathrm{A}_{02}+\ldots . .+\mathrm{A}_{0 \mathrm{i}}}
\end{aligned}
$$

In Equations 1 to 3:

a = sun radiation absorption, depending on the material and color of the exterior wall

$\mathrm{U}_{\mathrm{w}}=$ thermal transmittance of opaque wall (Watt $/ \mathrm{m}^{2} . \mathrm{K}$ ).

$\mathrm{WWR}=$ window-to-wall ratio $(\%)$

$\mathrm{TD}_{\mathrm{ek}}=$ equivalent temperature difference for wall ( $10^{\circ} \mathrm{K}$ for brick wall)

SC = shading coefficients of fenestrations, specified by the manufacturer

$\mathrm{SF}=$ solar factor $\left(\mathrm{W} / \mathrm{m}^{2}\right)$, depends on building orientation

$=130$ for North $(\mathrm{N}), 113$ for North East (NE), 112 for East (E), 97 for South East (SE), 97 for South (S), 176 for South West (SW), 243 for West (W), 211 for North West (NW)

$\mathrm{U}_{\mathrm{f}}=$ thermal transmittance of fenestration (Watt $\left./ \mathrm{m}^{2} .0 \mathrm{~K}\right)$

$\Delta \mathrm{T} \quad=$ temperature difference between exterior and interior design condition $\left(5^{\circ} \mathrm{K}\right)$

$\mathrm{A}_{1}=$ wall area with material $1\left(\mathrm{~m}^{2}\right)$

$\mathrm{A}_{2}=$ wall area with material $2\left(\mathrm{~m}^{2}\right)$

$\mathrm{An}=$ wall area with material $\mathrm{n} .\left(\mathrm{m}^{2}\right)$

$\Sigma \mathrm{A}=\mathrm{A} 1+\mathrm{A} 2+\ldots \ldots \ldots . . . \mathrm{An}$

$\mathrm{A}_{0 \mathrm{i}}=$ gross area of exterior wall 0i (opaque wall area + fenestration area). $\left(\mathrm{m}^{2}\right)$

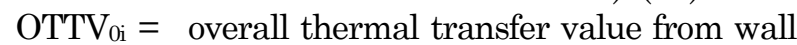
oi $\left(\mathrm{W} / \mathrm{m}^{2}\right)$

For the purpose of energy conservation, the maximum permissible OTTV is set to $35 \mathrm{Watt} / \mathrm{m}^{2}$.

\section{ETTV based on BCA of Singapore [5]}

Since 1979, the Building Control Regulations had prescribed an envelope thermal performance standard known as OTTV. The OTTV standard applied only to air-conditioned non-residential buildings. A major review of the OTTV formula was carried out in the early 2000 to provide a more accurate measure of the thermal performance of building envelope. The new formula is named Envelope Thermal Transfer Value (ETTV) to differentiate from the original OTTV formula. The ETTV requirement does not apply to non air-conditioned buildings.

The ETTV formulas are given as follows:

$$
\begin{aligned}
\mathrm{ETTV}= & 12(1-\mathrm{WWR}) \mathrm{U}_{\mathrm{w}}+3.4(\mathrm{WWR}) \mathrm{U}_{\mathrm{f}}+211 \\
(\mathrm{WWR})(\mathrm{CF})(\mathrm{SC}) & (4)
\end{aligned}
$$

To calculate the ETTVfor the envelope of the whole building, the following equation shall be used:

$$
\begin{aligned}
& \text { ETTV }= \\
& \frac{\left(\mathrm{A}_{01} \mathrm{ETTV}_{01}\right)+\left(\mathrm{A}_{02} \mathrm{ETTV}_{02}\right)+\ldots . .+\left(\mathrm{A}_{01} \mathrm{ETTV}_{0 \mathrm{i}}\right)}{\mathrm{A}_{01}+\mathrm{A}_{02}+\ldots . .+\mathrm{A}_{0 \mathrm{i}}}
\end{aligned}
$$

where:

ETTV = Envelope Thermal Transfer Value (Watt $\left./ \mathrm{m}^{2}\right)$

WWR = Window-to-Wall Ratio (Fenestration area/gross area of exterior wall) (\%)

$\mathrm{U}_{\mathrm{w}} \quad=$ Thermal transmittance of opaque wall (Watt $\left./ \mathrm{m}^{2} . \mathrm{K}\right)$

$\mathrm{U}_{\mathrm{f}} \quad=$ Thermal transmittance of fenestration $\left(\mathrm{Watt} / \mathrm{m}^{2} . \mathrm{K}\right)$

$\mathrm{CF} \quad=$ Correction Factor for solar heat gain through fenestration $\left(\mathrm{Watt} / \mathrm{m}^{2}\right)$, depends on pitch angle and orientation. Pitch angle is the numerical measurement of the slope of a wall. The pitch angle used for this study is $90^{\circ}$.

SC = Shading Coefficients of fenestration, specified by the manufacturer

$\mathrm{A}_{01}, \mathrm{~A}_{02}, \mathrm{~A}_{0 \mathrm{n}}=$ gross areas of the exterior wall for each orientation $\left(\mathrm{m}^{2}\right)$

For the purpose of energy conservation, the maximum permissible ETTV is set to $50 \mathrm{Watt} / \mathrm{m}^{2}$.

\section{Buildings Considered}

This study uses data and building specification from Singapore Reference Office Building Description [6]. The buildings that will be analyzed are hypothetical buildings with eight different building shapes and eight different building orientations.

In this study, the Authors calculated OTTV [3] and ETTV [5] of prismatic buildings with eight different shapes, eight building orientations, 11 Window to Wall Ratio (WWR), and 27 alternative for building envelope material for OTTV as shown in Table 1, and nine alternative for building envelope material for ETTV as shown in Table 2.

The Authors then compared the results to find the OTTV/ETTV value that fits the energy-saver building standard. The eight hypothetical building 
shapes; circular, equilateral octagon, equilateral triangle, parallelogram, square, rectangle, ellipse, and trapezoid with eight orientations (N, NE, E, SE, $\mathrm{S}, \mathrm{SW}, \mathrm{W}, \mathrm{NW}$ ) are shown in Figure 1. Each hypothetical building is ten story with four meters floor to floor height. The overall floor area is $625 \mathrm{~m}^{2}$ with $100 \mathrm{~m}^{2}$ core, leaving the total floor that requires cooling as $525 \mathrm{~m}^{2}$. Total area of the whole building is $5250 \mathrm{~m}^{2}$.

There are three materials considered for the building envelope.

1. Wall I: Aluminum Composite Panel $4 \mathrm{~mm}$, Aluminum Frame, Steel Bracket, and Gypsum Board $9 \mathrm{~mm}\left(\mathrm{U}_{\mathrm{wl}}=3.007 \mathrm{~W} / \mathrm{m}^{2} . \mathrm{K}\right)$

2. Wall II: Glass Fiber Reinforced Concrete 12.7 $\mathrm{mm}$, Steel Bracket, and Gypsum Board $9 \mathrm{~mm}$ $\left(\mathrm{U}_{\mathrm{wI}}=2.961 \mathrm{~W} / \mathrm{m}^{2} . \mathrm{K}\right)$

3. Wall III: Brick wall, plaster and ceramic tile 9 $\mathrm{mm}\left(\mathrm{U}_{\mathrm{wIII}}=2.741 \mathrm{~W} / \mathrm{m}^{2} . \mathrm{K}\right)$
For outdoor painting, the Authors chose three different colors to represent: the largest $\alpha$, overall black painting $\left(\alpha_{1}=0.95\right)$, the medium $\alpha$, medium green or blue painting $\left(\alpha_{2}=0.57\right)$, and the smallest $\alpha$, white varnish $\left(\alpha_{3}=0.21\right)$

For single glazed window, the Authors used high shading coefficient of $\mathrm{SC}_{\mathrm{H}}=0.88, \mathrm{U}_{\mathrm{fH}}=3.069$ $\mathrm{W} / \mathrm{m}^{2} . \mathrm{K}$, medium shading coefficient $\mathrm{SCM}_{\mathrm{M}}=0.62$, $\mathrm{U}_{\mathrm{fM}}$ $=3.069 \mathrm{~W} / \mathrm{m}^{2} \cdot \mathrm{K}$, and low shading coefficient $\mathrm{SC}_{\mathrm{L}}=$ $0.37, \mathrm{U}_{\mathrm{fL}}=3.08 \mathrm{~W} / \mathrm{m}^{2} . \mathrm{K}$. Only one building material is used for each alternative envelope building. WWR started from $0 \%$ to $100 \%$ with $10 \%$ interval.

Table 1 shows 27 different alternative combinations of materials for building envelope in OTTV calculation (marked with " $\checkmark$ " in Table 1 ). On the other hand, there are only nine different alternative combinations of materials for building envelope in ETTV calculation because ETTV calculation does not include the variable $\alpha$ (Table 2).

Table 1. 27 Alternatives of Building Envelope Materials on OTTV

\begin{tabular}{|c|c|c|c|c|c|c|c|c|c|c|c|c|}
\hline & \multicolumn{3}{|c|}{$\alpha$} & \multicolumn{3}{|c|}{ Building Envelope } & \multicolumn{6}{|c|}{ Glass } \\
\hline & $\alpha_{1}$ & $\alpha_{2}$ & $\alpha_{3}$ & $\mathrm{U}_{\mathrm{wl}}$ & $\mathrm{U}_{\mathrm{wII}}$ & $\mathrm{U}_{\mathrm{wII}}$ & $\mathrm{U}_{\mathrm{fH}}$ & $\mathrm{U}_{\mathrm{fM}}$ & $\mathrm{U}_{\mathrm{fL}}$ & $\mathrm{S}_{\mathrm{CH}}$ & $\mathrm{ScM}$ & $\mathrm{S}_{\mathrm{CL}}$ \\
\hline & 0.95 & 0.57 & 0.21 & 3.007 & 2.961 & 2.741 & 3.063 & 3.069 & 3.08 & 0.88 & 0.62 & 0.37 \\
\hline Alternative 1 & $\checkmark$ & & & $\checkmark$ & & & $\checkmark$ & & & $\checkmark$ & & \\
\hline Alternative 2 & $\checkmark$ & & & $\checkmark$ & & & & $\checkmark$ & & & $\checkmark$ & \\
\hline Alternative 3 & $\checkmark$ & & & $\checkmark$ & & & & & $\checkmark$ & & & $\checkmark$ \\
\hline Alternative 4 & $\checkmark$ & & & & $\checkmark$ & & $\checkmark$ & & & $\checkmark$ & & \\
\hline Alternative 5 & $\checkmark$ & & & & $\checkmark$ & & & $\checkmark$ & & & $\checkmark$ & \\
\hline Alternative 6 & $\checkmark$ & & & & $\checkmark$ & & & & $\checkmark$ & & & $\checkmark$ \\
\hline Alternative 7 & $\checkmark$ & & & & & $\checkmark$ & $\checkmark$ & & & $\checkmark$ & & \\
\hline Alternative 8 & $\checkmark$ & & & & & $\checkmark$ & & $\checkmark$ & & & $\checkmark$ & \\
\hline Alternative 9 & $\checkmark$ & & & & & $\checkmark$ & & & $\checkmark$ & & & $\checkmark$ \\
\hline Alternative 10 & & $\checkmark$ & & $\checkmark$ & & & $\checkmark$ & & & $\checkmark$ & & \\
\hline Alternative 11 & & $\checkmark$ & & $\checkmark$ & & & & $\checkmark$ & & & $\checkmark$ & \\
\hline Alternative 12 & & $\checkmark$ & & $\checkmark$ & & & & & $\checkmark$ & & & $\checkmark$ \\
\hline Alternative 13 & & $\checkmark$ & & & $\checkmark$ & & $\checkmark$ & & & $\checkmark$ & & \\
\hline Alternative 14 & & $\checkmark$ & & & $\checkmark$ & & & $\checkmark$ & & & $\checkmark$ & \\
\hline Alternative 15 & & $\checkmark$ & & & $\checkmark$ & & & & $\checkmark$ & & & $\checkmark$ \\
\hline Alternative 16 & & $\checkmark$ & & & & $\checkmark$ & $\checkmark$ & & & $\checkmark$ & & \\
\hline Alternative 17 & & $\checkmark$ & & & & $\checkmark$ & & $\checkmark$ & & & $\checkmark$ & \\
\hline Alternative 18 & & $\checkmark$ & & & & $\checkmark$ & & & $\checkmark$ & & & $\checkmark$ \\
\hline Alternative 19 & & & $\checkmark$ & $\checkmark$ & & & $\checkmark$ & & & $\checkmark$ & & \\
\hline Alternative 20 & & & $\checkmark$ & $\checkmark$ & & & & $\checkmark$ & & & $\checkmark$ & \\
\hline Alternative 21 & & & $\checkmark$ & $\checkmark$ & & & & & $\checkmark$ & & & $\checkmark$ \\
\hline Alternative 22 & & & $\checkmark$ & & $\checkmark$ & & $\checkmark$ & & & $\checkmark$ & & \\
\hline Alternative 23 & & & $\checkmark$ & & $\checkmark$ & & & $\checkmark$ & & & $\checkmark$ & \\
\hline Alternative 24 & & & $\checkmark$ & & $\checkmark$ & & & & $\checkmark$ & & & $\checkmark$ \\
\hline Alternative 25 & & & $\checkmark$ & & & $\checkmark$ & $\checkmark$ & & & $\checkmark$ & & \\
\hline Alternative 26 & & & $\checkmark$ & & & $\checkmark$ & & $\checkmark$ & & & $\checkmark$ & \\
\hline Alternative 27 & & & $\checkmark$ & & & $\checkmark$ & & & $\checkmark$ & & & $\checkmark$ \\
\hline
\end{tabular}


Table 2. Nine Alternatives of Building Envelope Materials on ETTV

\begin{tabular}{|c|c|c|c|c|c|c|c|c|c|}
\hline & \multicolumn{3}{|c|}{ Building Envelope } & \multicolumn{6}{|c|}{ Glass } \\
\hline & $\mathrm{U}_{\mathrm{wI}}$ & $\mathrm{U}_{\mathrm{wII}}$ & $\mathrm{U}_{\mathrm{wIII}}$ & $\mathrm{U}_{\mathrm{fH}}$ & $\mathrm{U}_{\mathrm{fM}}$ & $\mathrm{U}_{\mathrm{fL}}$ & $\mathrm{S}_{\mathrm{CH}}$ & $\mathrm{S}_{\mathrm{CM}}$ & $\mathrm{S}_{\mathrm{CL}}$ \\
\hline & 3.007 & 2.961 & 2.741 & 3.063 & 3.069 & 3.08 & 0.88 & 0.62 & 0.37 \\
\hline Alternative 1 & $\checkmark$ & & & $\checkmark$ & & & $\checkmark$ & & \\
\hline Alternative 2 & $\checkmark$ & & & & $\checkmark$ & & & $\checkmark$ & \\
\hline Alternative 3 & $\checkmark$ & & & & & $\checkmark$ & & & $\checkmark$ \\
\hline Alternative 4 & & $\checkmark$ & & $\checkmark$ & & & $\checkmark$ & & \\
\hline Alternative 5 & & $\checkmark$ & & & $\checkmark$ & & & $\checkmark$ & \\
\hline Alternative 6 & & $\checkmark$ & & & & $\checkmark$ & & & $\checkmark$ \\
\hline Alternative 7 & & & $\checkmark$ & $\checkmark$ & & & $\checkmark$ & & \\
\hline Alternative 8 & & & $\checkmark$ & & $\checkmark$ & & & $\checkmark$ & \\
\hline Alternative 9 & & & $\checkmark$ & & & $\checkmark$ & & & $\checkmark$ \\
\hline
\end{tabular}
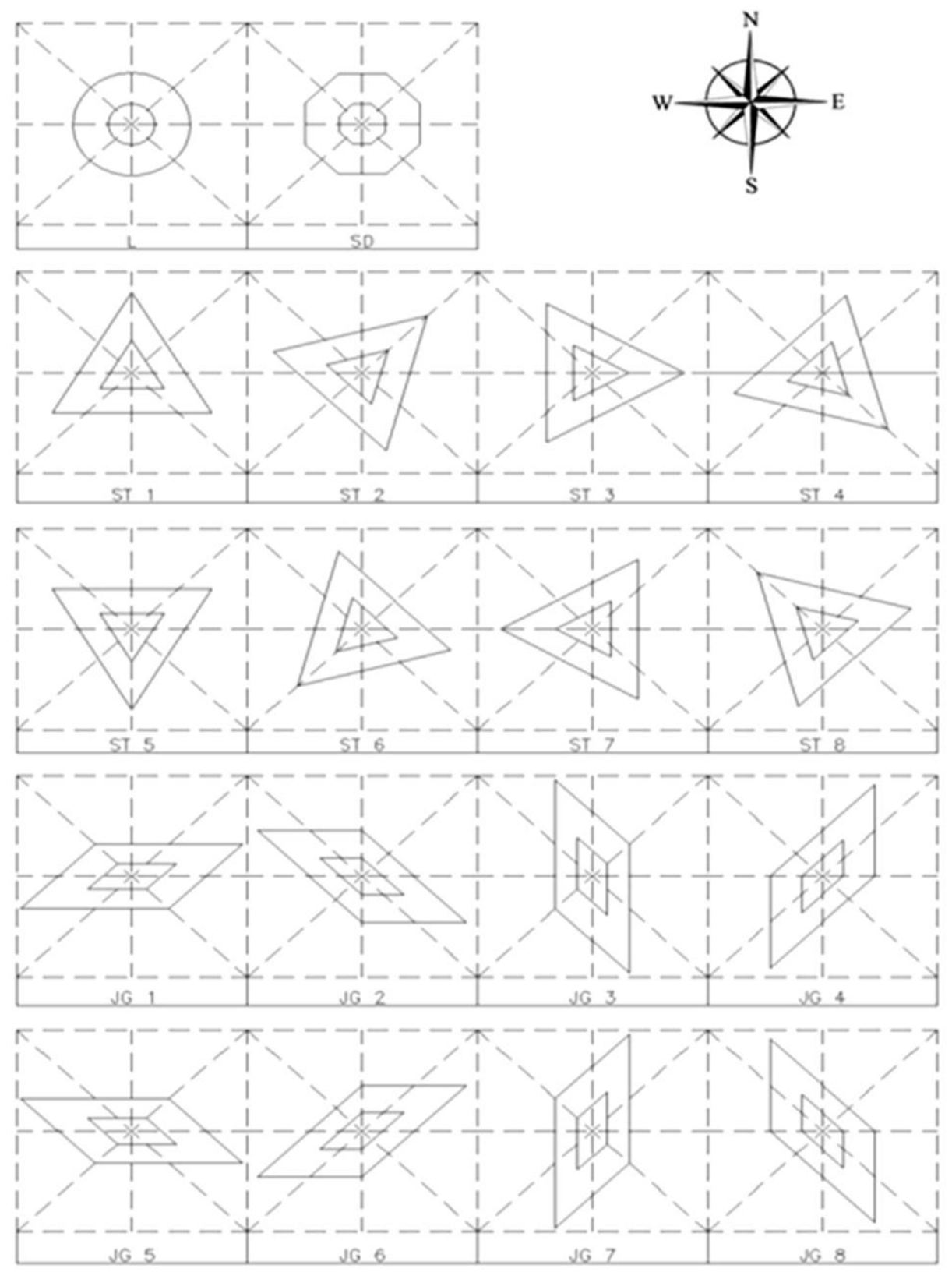

Figure 1.a. Four Building Shapes (Circular, Equilateral Octagon Equilateral Triangle, and Parallelogram) and Eight Building Orientations [7] 


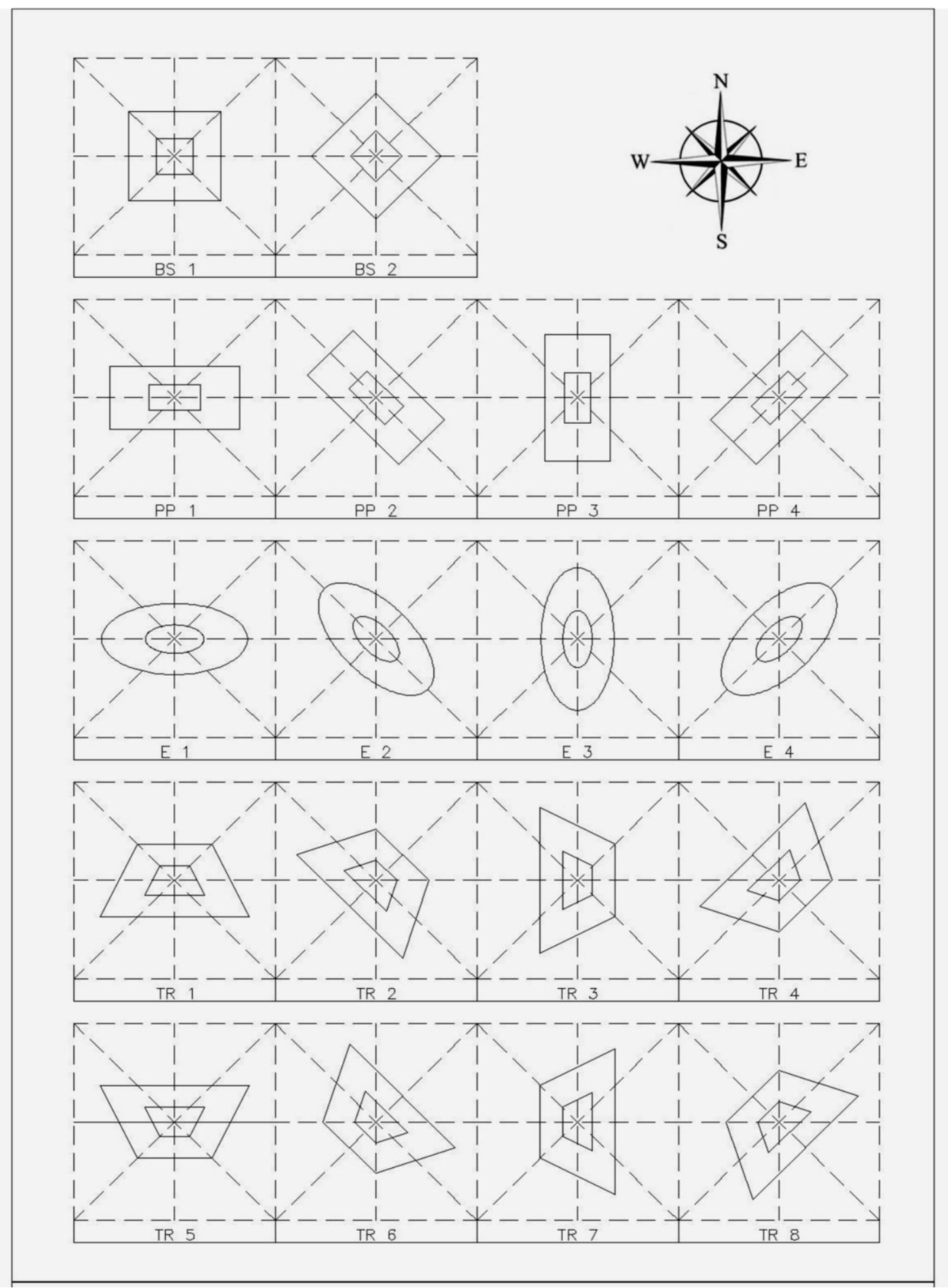

Figure 1.b. Four Building Shapes (Square, Rectangle, Ellipse, Trapezoid) and Eight Building Orientations [8]

\section{OTTV Calculation}

Table 3 shows a typical result of OTTV calculation on prismatic building, with circular shaped [7] using27 different materials and WWR from $0 \%$ to $100 \%$ with $10 \%$ interval. The shaded cells denote buildings with OTTV satisfying SNI 03-6389-2011

\section{ETTV Calculation}

Table 4 shows a typical result of ETTV calculation on prismatic building, with circular shape. This building was built with nine different materials with WWR from $0 \%$ to $100 \%$ with $10 \%$ interval. The shaded cells denote buildings with ETTV satisfying BCA 2008 
Table 3. OTTV for Prismatic Building with Circular Shape (Alt 1-27)

\begin{tabular}{|c|c|c|c|c|c|c|c|c|c|}
\hline WWR (\%) & Alt 1 & Alt 2 & Alt 3 & Alt 4 & Alt 5 & Alt 6 & Alt 7 & Alt 8 & Alt 9 \\
\hline 0 & 28.57 & 28.57 & 28.57 & 28.13 & 28.13 & 28.13 & 26.04 & 26.04 & 26.04 \\
\hline 10 & 40.21 & 36.38 & 32.70 & 39.82 & 35.99 & 32.31 & 37.94 & 34.11 & 30.43 \\
\hline 20 & 51.85 & 44.20 & 36.84 & 51.50 & 43.85 & 36.49 & 49.83 & 42.18 & 34.82 \\
\hline 30 & 63.50 & 52.01 & 40.98 & 63.19 & 51.71 & 40.67 & 61.73 & 50.24 & 39.21 \\
\hline 40 & 75.14 & 59.83 & 45.11 & 74.88 & 59.56 & 44.85 & 73.63 & 58.31 & 43.60 \\
\hline 50 & 86.79 & 67.64 & 49.25 & 86.57 & 67.42 & 49.03 & 85.52 & 66.38 & 47.98 \\
\hline 60 & 98.43 & 75.46 & 53.38 & 98.25 & 75.28 & 53.21 & 97.42 & 74.45 & 52.37 \\
\hline 70 & 110.07 & 83.27 & 57.52 & 109.94 & 83.14 & 57.39 & 109.32 & 82.51 & 56.76 \\
\hline 80 & 121.72 & 91.09 & 61.66 & 121.63 & 91.00 & 61.57 & 121.21 & 90.58 & 61.15 \\
\hline 90 & 133.36 & 98.90 & 65.79 & 133.32 & 98.86 & 65.75 & 133.11 & 98.65 & 65.54 \\
\hline 100 & 145.01 & 106.72 & 69.93 & 145.01 & 106.72 & 69.93 & 145.01 & 106.72 & 69.93 \\
\hline WWR (\%) & Alt 10 & Alt 11 & Alt 12 & Alt 13 & Alt 14 & Alt 15 & Alt 16 & Alt 17 & Alt 18 \\
\hline 0 & 17.14 & 17.14 & 17.14 & 16.88 & 28.13 & 16.88 & 15.62 & 15.62 & 15.62 \\
\hline 10 & 29.93 & 26.10 & 22.42 & 29.69 & 35.99 & 22.18 & 28.56 & 24.73 & 21.05 \\
\hline 20 & 42.71 & 35.06 & 27.70 & 42.50 & 43.85 & 27.49 & 41.50 & 33.84 & 26.48 \\
\hline 30 & 55.50 & 44.01 & 32.98 & 55.32 & 51.71 & 32.79 & 54.44 & 42.95 & 31.92 \\
\hline 40 & 68.29 & 52.97 & 38.26 & 68.13 & 59.56 & 38.10 & 67.38 & 52.06 & 37.35 \\
\hline 50 & 81.07 & 61.93 & 43.53 & 80.94 & 67.42 & 43.40 & 80.31 & 61.17 & 42.78 \\
\hline 60 & 93.86 & 70.89 & 48.81 & 93.75 & 75.28 & 48.71 & 93.25 & 70.28 & 48.21 \\
\hline 70 & 106.65 & 79.84 & 54.09 & 106.57 & 83.14 & 54.01 & 106.19 & 79.39 & 53.64 \\
\hline 80 & 119.43 & 88.80 & 59.37 & 119.38 & 91.00 & 59.32 & 119.13 & 88.50 & 59.07 \\
\hline 90 & 132.22 & 97.76 & 64.65 & 132.19 & 98.86 & 64.62 & 132.07 & 97.61 & 64.50 \\
\hline 100 & 145.01 & 106.72 & 69.93 & 145.01 & 106.72 & 69.93 & 145.01 & 106.72 & 69.93 \\
\hline WWR (\%) & Alt 19 & Alt 20 & Alt 21 & Alt 22 & Alt 23 & Alt 24 & Alt 25 & Alt 26 & Alt 27 \\
\hline 0 & 6.31 & 6.31 & 6.31 & 6.22 & 6.22 & 6.22 & 5.76 & 5.76 & 5.76 \\
\hline 10 & 20.18 & 16.35 & 12.68 & 20.10 & 16.27 & 12.59 & 19.68 & 15.85 & 12.17 \\
\hline 20 & 34.05 & 26.40 & 19.04 & 33.98 & 26.32 & 18.96 & 33.61 & 25.95 & 18.59 \\
\hline 30 & 47.92 & 36.44 & 25.40 & 47.85 & 36.37 & 25.33 & 47.53 & 36.04 & 25.01 \\
\hline 40 & 61.79 & 46.48 & 31.76 & 61.73 & 48.42 & 31.70 & 61.46 & 46.14 & 31.43 \\
\hline 50 & 75.66 & 56.52 & 38.12 & 75.61 & 56.47 & 38.07 & 75.38 & 56.24 & 37.84 \\
\hline 60 & 89.53 & 66.56 & 44.48 & 89.49 & 66.52 & 44.44 & 89.31 & 66.33 & 44.26 \\
\hline 70 & 103.40 & 76.60 & 50.84 & 103.37 & 76.57 & 50.82 & 103.23 & 76.43 & 50.68 \\
\hline 80 & 117.27 & 86.64 & 57.21 & 117.25 & 86.62 & 57.19 & 117.16 & 86.53 & 57.09 \\
\hline 90 & 131.14 & 96.68 & 63.57 & 131.13 & 86.67 & 63.56 & 131.08 & 96.62 & 63.51 \\
\hline 100 & 145.01 & 106.72 & 69.93 & 145.01 & 106.72 & 69.93 & 145.01 & 106.72 & 69.93 \\
\hline
\end{tabular}

OTTV satisfying the requirement of SNI 03-6389-2011

Table 4. ETTV for Prismatic Building with Circular Shape (Alt 1- 9)

\begin{tabular}{cccccccccc}
\hline WWR (\%) & \multicolumn{1}{c}{ Alt 1 } & \multicolumn{1}{c}{ Alt 2 } & \multicolumn{1}{c}{ Alt 3 } & \multicolumn{1}{c}{ Alt 4 } & \multicolumn{1}{c}{ Alt 5 } & Alt 6 & \multicolumn{1}{c}{ Alt 7 } & \multicolumn{1}{c}{ Alt 8 } & \multicolumn{1}{c}{ Alt 9 } \\
\hline 0 & 36.08 & 36.08 & 36.08 & 35.53 & 35.53 & 35.53 & 32.89 & 32.89 & 32.89 \\
10 & 52.15 & 46.65 & 41.36 & 51.66 & 46.15 & 40.86 & 49.28 & 43.78 & 38.49 \\
20 & 68.32 & 57.22 & 46.63 & 67.78 & 56.77 & 46.19 & 65.67 & 54.66 & 44.08 \\
30 & 84.30 & 67.78 & 51.91 & 83.91 & 67.40 & 51.52 & 82.06 & 65.55 & 49.67 \\
40 & 100.37 & 78.35 & 57.18 & 100.04 & 78.02 & 56.85 & 98.45 & 76.43 & 55.27 \\
50 & 116.44 & 88.91 & 62.46 & 116.16 & 88.64 & 62.18 & 114.84 & 87.32 & 60.86 \\
60 & 132.51 & 99.48 & 67.73 & 132.29 & 99.26 & 67.51 & 131.23 & 98.20 & 66.46 \\
70 & 148.58 & 110.05 & 73.01 & 148.41 & 109.88 & 72.84 & 147.62 & 109.09 & 72.05 \\
80 & 164.65 & 120.61 & 78.28 & 164.54 & 120.50 & 78.17 & 164.01 & 119.97 & 77.65 \\
90 & 180.72 & 131.18 & 83.56 & 180.66 & 131.12 & 83.50 & 180.40 & 130.86 & 83.24 \\
100 & 196.79 & 141.75 & 88.83 & 196.79 & 141.75 & 88.83 & 196.79 & 141.75 & 88.83 \\
\hline
\end{tabular}


Table 3 shows that for circular building the minimum WWR (WWRmin) that can satisfy the OTTV requirement is $10 \%$ with building envelope material of Alt 3, 6, 8-27, while the maximum WWR (WWRmax) is $40 \%$ with building envelope material of Alt 21, 24, 27. Through interpolation, the Authors determined that the WWRmin satisfying the OTTV requirement (35 Watt $/ \mathrm{m}^{2}$ ) as $5.52 \%$ with building envelope material of Alt 1, while the result of WWRmax is $45.57 \%$ with building envelope material of Alt 27.

Table 4 shows that for circular building the minimum WWR that can satisfy the ETTV requirement is $10 \%$ with building envelope material of Alt 2, 5, 7, 8 while the maximum WWR is $30 \%$ with building envelope material of Alt 9 . WWRmin satisfying the ETTV requirement (50 Watt $/ \mathrm{m}^{2}$ ) is $8.66 \%$, with building envelope material of Alt 1, while the result of WWRmax is $30.59 \%$, with building envelope material of Alt 9.

Interpolating other OTTV and ETTV for the rest of the buildings gives WWRmin and WWRmax as presented in Table 5.

Based on Table 5, the smallest WWR value for building material Alt $1\left(\alpha_{1}=0.95, \mathrm{U}_{\mathrm{wI}}=3.007, \mathrm{U}_{\mathrm{fH}}=\right.$ 3.063, $\left.\mathrm{SC}_{\mathrm{H}}=0.88\right)$ that satisfies OTTV requirements is Trapezoid TR7s $(W W R=4.66 \%)$. With the same building material $\left(\mathrm{U}_{\mathrm{wI}}=3.007, \mathrm{U}_{\mathrm{fH}}=3.063, \mathrm{SC}_{\mathrm{H}}=\right.$ 0.88), this WWRvalue is smaller than the WWR value that satisfied ETTV requirements (WWR = $7.69 \%$ ). This phenomena was influenced by the inclusion of the largest $\alpha$ value black-painted on OTTV formula.

On the other hand, the largest WWR value that satisfies OTTV requirements could be seen in parallelogram JG5 (WWR $=51.86 \%$ ) that used building material Alt $27\left(\alpha_{3}=0.21, \mathrm{U}_{\mathrm{wIII}}=2.741, \mathrm{U}_{\mathrm{fL}}=\right.$ $\left.3.08, \mathrm{SC}_{\mathrm{L}}=0.37\right)$. For the same building material $\left(\mathrm{U}_{\mathrm{wIII}}=2.741, \mathrm{U}_{\mathrm{fL}}=3.08, \mathrm{SC}_{\mathrm{L}}=0.37\right), \mathrm{WWR}$ value (WWR $=35.89 \%$ ) that satisfies ETTV requirement is smaller than $51.86 \%$.

The Authors also calculated the WWR value for building material Alt $10\left(\alpha_{2}=0.57, \mathrm{U}_{\mathrm{wI}}=3.007, \mathrm{U}_{\mathrm{fH}}=\right.$ 3.063, $\left.\mathrm{SC}_{\mathrm{H}}=0.88\right)$. The WWR value that satisfied OTTV requirement for parallelogram JG5 is $16.33 \%$, while the WWR value that satisfied ETTV requirement is $9.87 \%$.

The comparison of WWR maximum that satisfied OTTV and ETTV requirement is presented in Table 6.
Table 5. WWRmin and WWRmax (\%) Satisfying OTTV and ETTV Requirements

\begin{tabular}{|c|c|c|c|c|}
\hline & \multicolumn{2}{|c|}{ OTTV $=35 \mathrm{Watt} / \mathrm{m}^{2}$} & \multicolumn{2}{|c|}{$\mathrm{ETTV}=50 \mathrm{Watt} / \mathrm{m}^{2}$} \\
\hline & $\begin{array}{l}\text { Material } \\
\text { (alt 1) }\end{array}$ & $\begin{array}{c}\text { Material } \\
\text { (alt 27) }\end{array}$ & $\begin{array}{l}\text { Material } \\
\text { (alt 1) }\end{array}$ & $\begin{array}{c}\text { Material } \\
\text { (alt 9) }\end{array}$ \\
\hline & $\begin{array}{l}\text { WWR min } \\
(\%)\end{array}$ & $\begin{array}{c}\text { WWR max } \\
(\%)\end{array}$ & $\begin{array}{c}\text { WWR min } \\
(\%)\end{array}$ & $\begin{array}{c}\text { WWR max } \\
(\%)\end{array}$ \\
\hline Circular & 5.52 & 45.57 & 8.66 & 30.59 \\
\hline Octagon & 5.52 & 45.57 & 8.66 & 30.59 \\
\hline \multicolumn{5}{|l|}{ Triangle } \\
\hline ST1 & 5.83 & 47.50 & 9.31 & 33.40 \\
\hline ST2 & 5.88 & 47.79 & 8.73 & 30.88 \\
\hline ST3 & 5.38 & 44.64 & 8.13 & 28.36 \\
\hline ST4 & 5.85 & 47.59 & 8.73 & 30.88 \\
\hline ST5 & 6.13 & 49.28 & 9.27 & 33.22 \\
\hline ST6 & 5.38 & 44.64 & 8.60 & 30.32 \\
\hline ST7 & 5.39 & 44.72 & 8.01 & 27.88 \\
\hline ST8 & 5.16 & 43.25 & 8.66 & 30.59 \\
\hline \multicolumn{5}{|c|}{ Parallelogram } \\
\hline JG1 & 6.34 & 50.55 & 9.92 & 36.13 \\
\hline JG2 & 6.27 & 50.10 & 9.44 & 33.98 \\
\hline JG3 & 4.91 & 41.62 & 7.70 & 26.58 \\
\hline JG4 & 4.92 & 41.64 & 7.98 & 27.73 \\
\hline JG5 & 6.57 & 51.86 & 9.87 & 35.89 \\
\hline JG6 & 5.98 & 48.41 & 9.51 & 34.29 \\
\hline JG7 & 4.79 & 40.78 & 7.73 & 26.71 \\
\hline JG8 & 5.11 & 42.90 & 7.93 & 27.52 \\
\hline \multicolumn{5}{|l|}{ Square } \\
\hline BS1 & 5.60 & 46.06 & 8.72 & 30.85 \\
\hline BS2 & 5.44 & 45.12 & 8.60 & 30.32 \\
\hline \multicolumn{5}{|l|}{ Rectangle } \\
\hline PP1 & 6.10 & 49.13 & 9.39 & 33.75 \\
\hline PP2 & 5.51 & 45.49 & 8.58 & 30.25 \\
\hline PP3 & 5.18 & 43.37 & 8.15 & 28.42 \\
\hline PP4 & 5.39 & 44.68 & 8.61 & 30.39 \\
\hline \multicolumn{5}{|l|}{ Ellipse } \\
\hline $\mathrm{E} 1$ & 5.31 & 44.18 & 8.36 & 29.30 \\
\hline E2 & 5.49 & 45.35 & 8.67 & 30.61 \\
\hline E3 & 5.77 & 47.07 & 8.99 & 31.98 \\
\hline E4 & 5.56 & 45.77 & 8.65 & 30.54 \\
\hline \multicolumn{5}{|l|}{ Trapezoid } \\
\hline TR1 & 6.30 & 50.31 & 9.88 & 35.95 \\
\hline TR2 & 5.24 & 43.76 & 8.51 & 29.93 \\
\hline TR3 & 5.07 & 42.70 & 7.78 & 26.89 \\
\hline TR4 & 5.02 & 42.29 & 8.56 & 30.14 \\
\hline TR5 & 6.56 & 51.81 & 9.84 & 35.77 \\
\hline TR6 & 5.74 & 46.88 & 8.68 & 30.65 \\
\hline TR7 & 4.66 & 39.95 & 7.69 & 26.54 \\
\hline TR8 & 5.89 & 47.87 & 8.65 & 30.55 \\
\hline
\end{tabular}

Table 6. The Value of WWR $\mathrm{Wax}_{\text {ax }}$ that Satisfies OTTV/ETTV Requirement for Energy Efficient

\begin{tabular}{lcc}
\hline \multirow{2}{*}{ Building Shape } & \multicolumn{2}{c}{ WWR $_{\max }$} \\
\cline { 2 - 3 } & OTTV (Alt 27) & ETTV (Alt 9) \\
\hline Circular & $45.57 \%$ & $30.59 \%$ \\
Equilateral Octagon & $45.57 \%$ & $30.59 \%$ \\
Equilateral Triangle & $49.28 \%$ & $33.40 \%$ \\
Parallelogram & $51.86 \%$ & $36.13 \%$ \\
Square & $46.06 \%$ & $30.85 \%$ \\
Rectangle & $49.13 \%$ & $33.75 \%$ \\
Ellipse & $47.07 \%$ & $31.98 \%$ \\
Trapezoid & $51.81 \%$ & $35.95 \%$ \\
\hline
\end{tabular}




\section{Conclusions}

The requirements of ETTV for outdoor paintings with medium $\alpha\left(\alpha_{2}\right)$ and the smallest $\alpha\left(\alpha_{3}\right)$ are stricter than OTTV requirement. But for outdoor painting with the largest $\alpha\left(\alpha_{1}\right)$, OTTV has stricter regulation than ETTV.

From this study, the Authors concluded that the Parallelogram shape building with building orientation JG5/JG1 is the best combination of energy saver building. This combination created the biggest WWR value that satisfies the requirement of OTTV/ETTV regulation.

This study also highlighted the need for further analysis to determine the $\alpha$ value needed for creating a stricter ETTV value.

\section{References}

1. Koo, T.K., A Study on The Feasibility of Using The Overall Thermal Transfer Value (OTTV) on Assessing The Use of Building Energy in Hongkong, The Hong Kong Polytechnic University, 2001. http://www.worldenergy.org/wec-geis/wec_ congress/default.asp

2. Standar Nasional Indonesia (SNI) 03-6389-2000, Konservasi Energi Selubung Bangunan pada
Bangunan Gedung, Badan Standardisasi Nasional (BSN) Jakarta, Indonesia, 2000.

3. Standar Nasional Indonesia (SNI) 03-6389-2011, Konservasi Energi Selubung Bangunan pada Bangunan Gedung, Badan Standardisasi Nasional (BSN) Jakarta, Indonesia, 2011.

4. The Development \& Building Control Division (PWD), Handbook on Energy Conservation in Building and Building Services, Singapore, 1983.

5. Building and Construction Authority (BCA), Code on Envelope Thermal Performance for Buildings, Singapore, 2008.

6. The Development \& Building Control Division (PWD), Handbook on Energy Conservation in Building and Building Services, Singapore, 1975.

7. Prayoga, M.H., Analisa Bentuk, Orientasi, Selubung, Material Bangunan Berdasarkan RSNI 03-6389-2011 dan ETTV BCA 2008, Studi Kasus: Lingkaran, Segi Delapan Sama Sisi, Segitiga Sama Sisi, dan Jajaran Genjang, Graduate Thesis, Petra Christian University, Surabaya, Indonesia, 2011 (in Indonesian).

8. Gunawan, Y.P., Analisa Bentuk, Orientasi, Selubung, Material Bangunan Berdasarkan RSNI 03-6389-2011 dan ETTV BCA 2008, Studi Kasus: Bujur Sangkar, Persegi Panjang, Elips, dan Trapesium, Graduate Thesis, Petra Christian University, Surabaya, Indonesia, 2011 (in Indonesian). 